\title{
EIGENMAPS OF DYNAMIC FUNCTIONAL CONNECTIVITY: VOXEL-LEVEL DOMINANT PATTERNS THROUGH EIGENVECTOR CENTRALITY
}

\author{
Maria Giulia Preti ${ }^{1,2}$ and Dimitri Van De Ville ${ }^{1,2}$ \\ ${ }^{1}$ Institute of Bioengineering, École Polytechnique Fédérale de Lausanne (EPFL), Switzerland \\ ${ }^{2}$ Faculty of Medicine, University of Geneva, Switzerland
}

\begin{abstract}
Dynamic functional connectivity $(\mathrm{dFC})$ based on resting-state functional magnetic resonance imaging (fMRI) explores the ongoing temporal configuration of brain networks. To reduce the large dimensionality of the data, conventional dFC analysis usually foresees an atlasing step, in which the brain is parcellated into specific regions of interest, and voxels' timecourses are spatially averaged within these regions before assessing connectivity. In this study, we addressed for the first time the exploration of $\mathrm{dFC}$ at the voxel level; i.e., without the use of any brain parcellation prior to the connectivity analysis. We used a sliding-window approach and extracted window-specific dominant patterns. To overcome the limitations due to the huge size of voxelwise connectivity matrices, we adopted the fast eigenvector centrality method with some adaptations to make it suitable for the dFC framework. After concatenation of the dominant patterns of all subjects, principal component analysis (PCA) was used to extract the eigenmaps; i.e., the most recurring voxelwise brain patterns characterizing resting-state. The obtained eigenmaps appeared consistent with previously observed resting-state eigenconnectivities, but with the substantial advantage of characterizing brain networks at the voxel level without the need of an atlas. The effect of the connection-wise temporal demeaning, usually performed in $\mathrm{dFC}$ analysis to remove the influence of static connectivity, was explored and does not seem to have an influence when voxelwise brain patterns are targeted.
\end{abstract}

Index Terms - Voxelwise, functional imaging, connectivity analysis, fMRI analysis.

\section{INTRODUCTION}

Dynamic functional connectivity ( $\mathrm{dFC}$ ) based on resting-state functional magnetic resonance imaging (fMRI) aims at ex-

This work was supported by the Swiss National Science Foundation (grant 205321-163376), the Center for Biomedical Imaging (CIBM) of Geneva and Lausanne Universities and the Leenaards and Louis-Jeantet Foundations. Data were provided by the Human Connectome Project, WUMinn Consortium (Principal Investigators: David Van Essen and Kamil Ugurbil; 1U54MH091657) funded by the 16 NIH Institutes and Centers that support the NIH Blueprint for Neuroscience Research; and by the McDonnell Center for Systems Neuroscience at Washington University. ploring the configuration of brain networks and their rearrangement over time [1]. The fMRI timecourses are commonly divided into subsequent partially overlapping windows and connectivity is estimated for each of these temporal segments; in this way, the temporal variations of each connection along the time of the scan can be observed [2]. The collected and available information is extremely large, given that the fMRI acquisition yields a time course for every voxel in the brain, and that the connectivity between every pair of voxels in gray matter (GM) can theoretically be assessed. However, FC analysis at the voxel level remains to date computationally challenging, due to the difficulties of storing the large voxel-by-voxel connectivity matrix, which can easily reach the order of $10^{10}$ entries. This dimensionality problem appears even amplified when the exploration of the dynamical properties of connectivity is addressed, where several connectivity matrices — one for each window—should be computed and stored. Due to this limit, the connectivity analysis has been limited in most of cases so far to only a small portion of the data available, by converting the original dataset to a lower dimensionality one [3]. This is possible with seed-based approaches, where an a-priori assumption of where the effects of interest are located is required, or with parcellation-based approaches, where voxels are usually aggregated in regions of interest and the timecourses of voxels within the same region are spatially averaged before assessing connectivity. In this way, the dimensionality can be effectively reduced by a factor of $10^{6}\left(10^{4}\right.$ vs $10^{10}$ connections to be explored). However, atlas-based approaches are not fully data-driven and require the a priori definition of a brain parcellation, which could heavily influence the results of the analysis. The use of different parcellation schemes, in fact, could lead to substantially different network structure and statistics [4-6]. Further, there is no consensus on the ideal parcellation resolution, which should be fine enough to only aggregate voxels with homogeneous fMRI response, and at the same time coarse enough to preserve the anatomical interpretability of the segmented regions [6, 7]. Data-driven techniques like independent component analysis (ICA) overcome the problem of parcellation, but nevertheless require the a-priori choice of other critical parameters, such as the number of components to estimate, which influences the resulting network configurations 
[3]. So far, only few studies proposed a voxelwise exploration of functional connectivity properties [8-13], but always limiting the analysis to a static approach, therefore, providing a measure of the average functional connectivity within the acquisition time. Wink and colleagues [8] introduced and validated an efficient method to characterize the brain network at the voxel level by estimating a specific graphical property; i.e., eigenvector centrality, without the need of explicitly computing the voxelwise connectivity matrix. This computational advantage appears crucial when willing to apply the analysis to $\mathrm{dFC}$, where the storing of approximately $10^{3}$ voxelwise FC matrices could be required. With this approach in mind, we address here the problem of assessing dynamic functional connectivity features without the use of any brain parcellation. On a voxel basis, we aim to retrieve the dominant patterns of connectivity and their changes over time. These dominant patterns-networks that are mostly dominant to express the implicit dFC matrix — can be extracted in a dynamic fashion by computation of the eigenvector centrality for every temporal window. Afterwards, we concatenate the centralities of all windows and subjects and applied principal component analysis (PCA) to extract the mostly recurring voxel-wise dominant patterns characterizing the population (i.e., eigenmaps), which we can represent as brain maps. We applied this method to the analysis of 11 healthy subjects and extracted the group eigenmaps characterizing resting state.

\section{METHODS}

\subsection{Subjects}

$N_{s}=11$ healthy subjects from the Human Connectome Project (HCP) database [14] (www.humanconnectome.org) were selected and their resting-state functional (Gradientecho EPI, TR $=720 \mathrm{~ms}, \mathrm{TE}=33.1 \mathrm{~ms}$, flip angle $=52^{\circ}, \mathrm{FOV}$ $=[208 \times 180]$, voxel size $=2 \mathrm{~mm}$ isotropic $)$ and structural $(3 \mathrm{D}$ MPRAGE T1-weighted, TR $=2400 \mathrm{~ms}, \mathrm{TE}=2.14 \mathrm{~ms}, \mathrm{TI}=1000$ $\mathrm{ms}$, flip angle $=8^{\circ}, \mathrm{FOV}=[224 \times 224]$, voxel size $=0.7 \mathrm{~mm}$ isotropic) acquisitions were considered.

\subsection{MRI preprocessing}

Standard preprocessing [15] and registration to MNI standard space [16] had already been performed on the HCP images. Additionally, functional volumes were spatially smoothed by convolution with a Gaussian kernel (5mm FWHM) using SPM8 (FIL,UCL,UK). The first 10 volumes were discarded so that the fMRI signal achieves steady-state magnetization, resulting in $T=1190$ time points considered. The T1 image was linearly registered to the mean functional volume and individual tissue maps were segmented (white matter, gray matter, cerebrospinal fluid). Voxel timecourses were detrended and nuisance variables were regressed out (6 head motion parameters, average cerebrospinal fluid and white matter signal computed in standard masks mapped to the subject's fMRI space and masked with individual segmentation maps). Then, the preprocessed voxel time courses were bandpass filtered $([0.01-0.15 \mathrm{~Hz}])$ to focus on the resting-state fluctuations. Finally, a standard GM parcellation in MNI coordinates (IIT GM Destrieux mask, http://www.iit.edu/) was resliced to fMRI resolution and used to mask the fMRI volumes, in order to restrict the analysis to voxels belonging to GM and to consider the same locations for every subject (number of voxels $N_{v}=116.912$ ).

\subsection{Dominant connectivity patterns through dynamic eigenvector centrality}

Taking inspiration from the fast eigenvector centrality computation proposed by Wink and colleagues [8] for static functional connectivity, we propose here the following methodological framework to extract dynamic dominant connectivity patterns. Let $\mathbf{X}$ denote the $N_{v} \times T$ fMRI data matrix containing in its rows the fMRI preprocessed timecourses of voxels located in GM. The main idea of our method consists in adopting a sliding-window approach and computing the eigenvector centrality (i.e. the eigenvector corresponding to the largest eigenvalue) of the connectivity matrix for each window. Choosing window length $w=83$ TR (59.76s) and step $s=2$ TR (1.44s), the original matrix $\mathbf{X}$ was split into $N_{w}$ matrices $\boldsymbol{X}_{i}$ of size $N_{v} \times w$ to be analyzed separately. For each window $i=1, \ldots, N_{w}$, the eigenvector centrality of the FC matrix $\boldsymbol{C}_{i}=\boldsymbol{X}_{i} \boldsymbol{X}_{i}{ }^{\mathrm{T}}$ yields the dominant brain pattern for that specific time segment. However, as the direct assessment of $\boldsymbol{C}_{i}$ would be computationally extremely heavy, a specific strategy, detailed in the following, was adopted to estimate centralities keeping $\boldsymbol{C}_{i}$ implicit. Further, the following issues must be addressed in the $\mathrm{dFC}$ framework: 1) the matrices $\boldsymbol{X}_{i}$ should be specifically normalized so that every $C_{i}$ yields Pearson's correlation coefficients; 2) the demeaning of connection timecourses, which is normally performed in $\mathrm{dFC}$ analysis to focus exclusively on the FC fluctuations (and not on the static FC values) should be included.

For $C_{i}$ to yield Pearson's correlation coefficients, every row $\boldsymbol{x}_{i}{ }^{(\mathrm{j})}\left(j=1, \ldots, N_{v}\right)$ of the window matrix $\boldsymbol{X}_{i}$ was normalized as follows (Eq. (1)):

$$
\boldsymbol{x}_{i}{ }^{(\mathrm{j})} \rightarrow \frac{\operatorname{zscore}\left(\boldsymbol{x}_{i}{ }^{(\mathrm{j})}\right)}{\sqrt{w}} .
$$

Then, to remove the effect of static FC, we proposed to subtract the static FC matrix $\boldsymbol{C}=\boldsymbol{X} \boldsymbol{X}^{\mathrm{T}}$, derived from the full acquisition, from every $\boldsymbol{C}_{i}$. Prior to that, the rows of the full data matrix $\boldsymbol{X}$ also needed to be normalized as shown in Eq. (1), for $C$ to contain correlation values. However, as it happens for $\boldsymbol{C}_{i}$, the direct assessment of $\boldsymbol{C}$ is also computationally problematic due to its large size, therefore we proposed to adopt the following approach, allowing to approximate the matrix by using its first $N_{a p p}$ eigenvectors without the need 
of computing and storing it. The first $N_{a p p}$ eigenvectors $\boldsymbol{v}_{k}$ $\left(k=1, \ldots, N_{a p p}\right)$ and eigenvalues $\lambda_{k}$ of $\boldsymbol{C}$ can be found by solving the eigendecomposition problem in Eq. (2):

$$
\boldsymbol{C} \boldsymbol{v}_{k}=\lambda_{k} \boldsymbol{v}_{k}
$$

Eigenvectors and eigenvalues were estimated without the direct computation of $\boldsymbol{C}$ with the ARPACK software library implemented in Matlab. This was possible by factorizing the matrix $\boldsymbol{C}$ into $\boldsymbol{X} \boldsymbol{X}^{\mathrm{T}}$, and choosing the order of operations as shown in Eq. (3):

$$
\boldsymbol{X}\left(\boldsymbol{X}^{\mathrm{T}} \boldsymbol{v}_{k}\right)=\lambda_{k} \boldsymbol{v}_{k}
$$

In this way, we reduced the computation to matrix-vector products, without requiring the direct assessment of the large matrix $\boldsymbol{X} \boldsymbol{X}^{\mathrm{T}}$. Then, the matrix $\boldsymbol{C}$ was approximated using its $N_{a p p}$ first eigenvectors and eigenvalues as follows (Eq. (4)):

$$
\boldsymbol{C} \approx \sum_{k=1}^{N_{a p p}} \lambda_{k} \boldsymbol{v}_{k} \boldsymbol{v}_{k}^{\mathrm{T}}
$$

where $N_{a p p}$ was set to 50 . Once estimated $\boldsymbol{C}$, the dominant pattern of each window $i$ can be computed as eigenvector centrality $\boldsymbol{v}_{i, 1}^{\prime}$ of the matrix $\boldsymbol{C}_{i}-\boldsymbol{C}$. The same strategy described above (Eq. (2), (3)) was therefore adopted for the eigendecomposition problem in Eq. (5):

$$
\left(\boldsymbol{C}_{i}-\boldsymbol{C}\right) \boldsymbol{v}_{i, 1}^{\prime}=\lambda_{i, 1}^{\prime} \boldsymbol{v}_{i, 1}^{\prime}
$$

which after factorization of $\boldsymbol{C}_{i}$ into $\boldsymbol{X}_{i} \boldsymbol{X}_{i}{ }^{\mathrm{T}}$ can be written as (Eq. (6)):

$$
\boldsymbol{X}_{i}\left(\boldsymbol{X}_{i}^{\mathrm{T}} \boldsymbol{v}_{i, 1}^{\prime}\right)-\sum_{k=1}^{N_{a p p}} \lambda_{k} \boldsymbol{v}_{k}\left(\boldsymbol{v}_{k}^{\mathrm{T}} \boldsymbol{v}_{i, 1}^{\prime}\right)=\lambda_{i, 1}^{\prime} \boldsymbol{v}_{i, 1}^{\prime}
$$

Again, the problem is reduced here to matrix-vector products which do not require the explicit computation of the $N_{v} \times N_{v}$ connectivity matrices.

\subsection{Group-specific resting-state eigenmaps}

The eigenvector centralities $\boldsymbol{v}_{i, 1}^{\prime}$ were then aggregated across windows and subjects into the $N_{v} \times N_{w} N_{s}$ matrix $\boldsymbol{E}$, and PCA was applied by means of singular value decomposition (SVD) of $\boldsymbol{E}$, yielding (Eq. (7)):

$$
\boldsymbol{E}=\boldsymbol{U}_{\boldsymbol{E}} \boldsymbol{S}_{\boldsymbol{E}} \boldsymbol{V}_{\boldsymbol{E}}^{\mathrm{T}}
$$

The left singular vectors, i.e. the columns of $\boldsymbol{U}_{\boldsymbol{E}}$, represent the resting-state brain dominant patterns of the specific population, which characterize the brain at the voxel level and can be therefore referred to as eigenmaps.

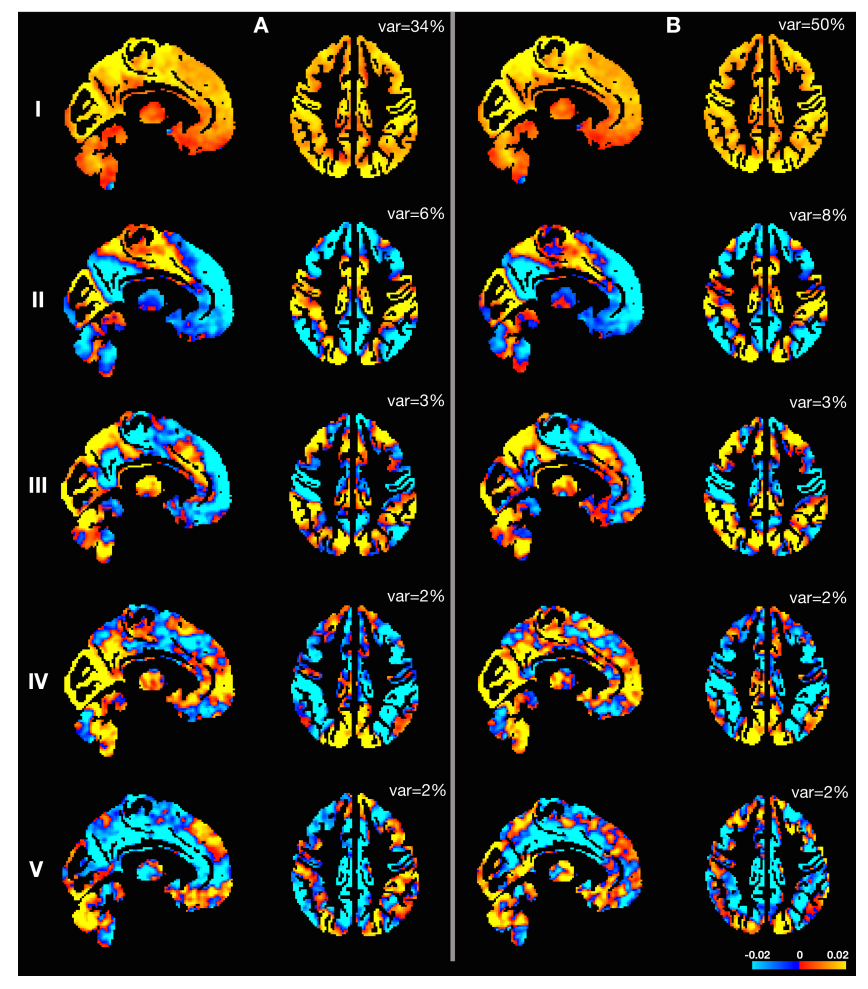

Fig. 1. First five eigenmaps obtained (A) with and (B) without connection-wise demeaning (sagittal and axial views).

FSLview was used as visualization tool. The percentage of variance explained by each component is reported.

\subsection{To demean or not to demean?}

To investigate the effect of the connection-wise temporal demeaning on the dominant brain patterns, we performed the same voxelwise analysis without demeaning as well. For each window $i$, the eigendecomposition problem was then reduced to (Eq. (8)):

$$
\boldsymbol{C}_{i} \boldsymbol{v}_{i, 1}^{\prime}=\lambda_{i, 1}^{\prime} \boldsymbol{v}_{i, 1}^{\prime}
$$

which was then solved similarly as before (Eq. (9)):

$$
\boldsymbol{X}_{i}\left(\boldsymbol{X}_{i}^{\mathrm{T}} \boldsymbol{v}_{i, 1}^{\prime}\right)=\lambda_{i, 1}^{\prime} \boldsymbol{v}_{i, 1}^{\prime}
$$

The eigenmaps obtained after PCA (Eq. (7)) with and without demeaning were then compared to explore if the temporal demeaning of connections alters the voxelwise dominant patterns.

\section{RESULTS AND DISCUSSION}

The 50 components used to approximate the full matrix $C$ explained $73 \%$ of the total variance. The first five restingstate eigenmaps derived from PCA decomposition explained respectively the $47 \%$ of the total variance when performing connection-wise demeaning (Fig. 1, A) and the 65\% without demeaning (Fig. 1, B). The effect of the demeaning converts 
the fluctuations around the static FC into positive and negative values. The similarity between the two sets of maps (Fig. 1) is striking, suggesting that the temporal demeaning, performed at the level of connections, does not affect the results at the voxel level in terms of dominant patterns. From this observation, we could infer that static FC values do not influence the analysis of brain dominant patterns, even if their effect is not explicitly removed. In addition, the temporal demeaning prior to eigendecomposition causes the loss of positive semidefinite property of $\boldsymbol{C}_{i}$, resulting in negative eigenvalues.

Concerning the networks highlighted by the resting-state eigenmaps, we can easily find a parallel with the patterns of the eigenconnectivities found previously [17]. The first map, i.e., the component explaining the largest variance in the data, consists of a global component which is also found in $\mathrm{dFC}$ eigenconnectivity analysis, and reflects fluctuations characterizing the whole brain. Interestingly, the second component clearly contrasts core regions of the default mode network (DMN) (posterior cingulate cortex, precuneus, angular gyrus, medial prefrontal cortex, shown in blue) with regions of the somatosensory cortex (superior parietal lobule, occipital visual areas, supplementary motor area, shown in red), in agreement with previous findings related to eigenconnectivities [17]. In the third eigenmap, memory-related regions (shown in blue), such as temporal cortex, hippocampus and parahippocampus, are identified in contrast with superior frontal regions, inferior parietal and supra marginal gyri (shown in red), again consistently with previous findings. The obtained eigenmaps proved therefore to successfully retrieve the networks involved in resting-state dynamics, having at the same time the advantage of being completely data-driven, without the need of any a-priori assumption on the data. Further, thanks to the strategies adopted for matrix eigendecomposition, results at the voxel-scale can be obtained without particular computational requirements.

\section{CONCLUSION}

We addressed here for the first time the observation of dynamic functional connectivity patterns at the voxel-scale, by means of extraction of dominant patterns in different temporal segments during the fMRI acquisition. Specific methodological strategies were adopted to make the analysis computationally possible without the need of reducing the dimensionality by using a brain parcellation. The analysis conducted on 11 healthy subjects allowed to retrieve group-specific restingstate eigenmaps in accordance with previously found and well-known resting-state networks. This approach opens new avenues for atlas-free $\mathrm{dFC}$.

\section{REFERENCES}

[1] C. Chang, G. Glover, "Time-frequency dynamics of resting-state brain connectivity measured with fMRI," NeuroImage, vol. 50, pp. 81-98, 2010.
[2] V. D. Calhoun, R. Miller, G. Pearlson, T. Adali, "The Chronnectome: Time-Varying Connectivity Networks as the Next Frontier in fMRI Data Discovery," Neuron, vol. 84, pp. 262-274, 2014.

[3] S. M. Rajtmajer, A. Roy, R. Albert, P. C. M. Molenaar, F. G. Hillary, "A voxelwise approach to determine consensus regionsof-interest for the study of brain network plasticity," Front Neuroanat, vol.9(97), 2015.

[4] J. Wang, L. Wang, Y. Zang, H. Yang, H. Tang, et al., "Parcellation-Dependent Small-World Brain Functional Networks: A Resting-State fMRI Study," Hum Brain Mapp, vol. 30, pp. 1511-1523, 2009.

[5] S. M. Smith, K. L. Miller, G. Salimi-Khorshidi, M. Webster, C. F. Beckmann, et al., "Network modelling methods for FMRI," NeuroImage, vol. 54, pp. 875-891, 2011.

[6] R. C. Craddock, G. A. James, P. E. Holtzheimer III, X. P. Hu, H. S. Mayberg, "A whole brain fMRI atlas generated via spatially constrained spectral clustering," Hum Brain Mapp, vol. 33(8), pp. 1914-28, 2012.

[7] A. Khazaee, A. Ebrahimzadeh, A. Babajani-Feremi, "Application of advanced machine learning methods on resting-state fMRI network for identification of mild cognitive impairment and Alzheimer?s disease," Brain Imaging Behav, In press, 2015.

[8] A. M. Wink, J. C. de Munck, Y. D. van der Werf, O. A. van den Heuvel, F. Barkhof, "Fast Eigenvector Centrality Mapping of Voxel-Wise Connectivity in Functional Magnetic Resonance Imaging: Implementation, Validation, and Interpretation," Brain Connect, vol. 2(5), pp. 265-274, 2012.

[9] S. Markett, C. Montag, B. Heeren, R. Saryiska, B. Lachmann, et al., "Voxelwise eigenvector centrality mapping of the human functional connectome reveals an influence of the catecholOmethyltransferase val158met polymorphism on the default mode and somatomotor network," Brain Struct Funct, In press.

[10] R. L. Buckner, J. Sepulcre, T. Talukdar, F. M. Krienen, H. Liu, et al., "Cortical Hubs Revealed by Intrinsic Functional Connectivity: Mapping, Assessment of Stability, and Relation to Alzheimer's Disease," J Neurosci, vol. 29(6), pp. 1860 ?1873, 2009.

[11] P. McCarthy, L. Benuskova, E. A. Franz, "The age-related posterior-anterior shift as revealed by voxelwise analysis of functional brain networks," Front Aging Neurosci, vol. 6, pp. 1-14, 2014.

[12] M. Markosova, L. Franz, L. Benuskov, "Topology of Brain Functional Networks: Towards the Role of Genes," ICONIP 2008, vol. part I, pp. 111-118, 2009.

[13] V. M. Eguiluz, D. R. Chialvo, G. A. Cecchi, M. Baliki, A. V. Apkarian, "Scale-Free Brain Functional Networks," Phys Rev Lett, vol. 94, pp. 018102-1-4, 2005.

[14] D. C. Van Essen, S. M. Smith, D. M. Barch, T. E. J. Behrens, E. Yacoub, K. Ugurbil, "The WU-Minn Human Connectome Project: An overview. NeuroImage," NeuroImage, vol. 80 , pp. 62-79, 2013.

[15] M. F. Glasser, S. N. Sotiropoulos, J. A. Wilson, T. S. Coalson, B. Fischl, et al., "The minimal preprocessing pipelines for the Human Connectome Project," Neurolmage, vol. 80, pp. 105-124, 2013.

[16] M. Jenkinson, P. R. Bannister, J. M. Brady, S. M. Smith, "Improved optimisation for the robust and accurate linear registration and motion correction of brain images," NeuroImage, vol. 17, pp. 825-841, 2002.

[17] N. Leonardi, J. Richiardi, M. Gschwind, S. Simioni, J.M. Annoni, et al., "Principal components of functional connectivity: a new approach to study dynamic brain connectivity during rest," Neuroimage, vol. 83, pp. 937-950, 2013. 